\title{
The mean square of the error term in a generalization of Dirichlet's divisor problem
}

by

\author{
Tom Meurman (Turku)
}

1. Introduction. Let $\sigma_{a}(n)$ denote the $n$th coefficient of the Dirichlet series $\zeta(s) \zeta(s-a)$, where $\zeta(s)$ is the Riemann zeta-function. Thus

We define

$$
\sigma_{a}(n)=\sum_{d \mid n} d^{a} .
$$

$$
D_{a}(y)=\sum_{n<y} \sigma_{a}(n)+\sigma_{a}(y) / 2
$$

with the convention that $\sigma_{a}(y)=0$ unless $y$ is an integer. We also define

$$
\Delta_{a}(y)=D_{a}(y)-\zeta(1-a) y-\frac{\zeta(1+a)}{1+a} y^{1+a}+\frac{1}{2} \zeta(-a) .
$$

In these definitions $a$ may be any complex number. We prove

Theorem. Suppose $x \geq 1$. Then

$$
\int_{1}^{x} \Delta_{a}(y)^{2} d y= \begin{cases}c_{1} x^{3 / 2+a}+O(x) & \text { for }-1 / 2<a<0 \\ c_{2} x \log x+O(x) & \text { for } a=-1 / 2, \\ O(x) & \text { for }-1<a<-1 / 2\end{cases}
$$

where

$$
\begin{aligned}
& c_{1}=(6+4 a)^{-1} \pi^{-2} \zeta(3 / 2-a) \zeta(3 / 2+a) \zeta(3 / 2)^{2} \zeta(3)^{-1}, \\
& c_{2}=\zeta(3 / 2)^{2} /(24 \zeta(3))
\end{aligned}
$$

and the constants implied by the $O$-symbols may depend on a.

This improves and generalizes a special case of a result of Kiuchi [3]. Kiuchi studied the situation in which $\sigma_{a}(n)$ is multiplied by $e^{2 \pi i n h / k}$, where $h$ and $k$ are coprime integers. In the case $k=1$ he proved that

$$
\int_{1}^{x} \Delta_{a}(y)^{2} d y=c_{1} x^{3 / 2+a}+O\left(x^{5 / 4+a / 2+\varepsilon}\right)
$$

for $-1 / 2<a<0$ and any positive $\varepsilon$. 
It is also interesting to record the situation in the case $a=0$. We have

$$
\Delta_{0}(y)=D_{0}(y)-y(\log y+2 \gamma-1)-1 / 4,
$$

where $\gamma$ is Euler's constant. Tong [11] proved that

$$
\int_{1}^{x} \Delta_{0}(y)^{2} d y=c_{0} x^{3 / 2}+O\left(x \log ^{5} x\right)
$$

where $c_{0}=\zeta(3 / 2)^{4} /\left(6 \pi^{2} \zeta(3)\right)$. A simpler proof of this was later given by Meurman [6], and subsequently Preissmann [9] improved the error term to $O\left(x \log ^{4} x\right)$.

Our theorem is analogous to what has been proved by Matsumoto and Meurman $[4,5]$ for $E_{(1-a) / 2}(T)$, the error term in the asymptotic formula for $\int_{1}^{T}|\zeta((1-a) / 2+i t)|^{2} d t$. However, in the special case $a=-1 / 2$ it is in fact sharper than what is suggested by the result just referred to.

The mean square estimates above show that the average order of $\Delta_{a}(y)$ is $O\left(y^{1 / 4+a / 2}\right)$ for $-1 / 2<a \leq 0, O(\sqrt{\log y})$ for $a=-1 / 2$ and $O(1)$ for $-1<$ $a<-1 / 2$. They also show that $\Delta_{a}(y)=\Omega\left(y^{1 / 4+a / 2}\right)$ for $-1 / 2<a \leq 0$ and $\Delta_{-1 / 2}(y)=\Omega(\sqrt{\log y})$. All this agrees with Pétermann's [8] conjecture (S) concerning individual values of $\Delta_{a}(y)$. As to the latter problem, a simple elementary argument starting with the definition of $\Delta_{a}(y)$ shows that $\Delta_{a}(y) \ll y^{(1+a) / 2}$. Pétermann [8] has a better result which is stated in terms of exponent pairs. Suffice it to say that it implies at least that $\Delta_{a}(y) \ll y^{(1+a) / 3+\varepsilon}$ for any positive $\varepsilon$. However, the true order of magnitude of $\Delta_{a}(y)$ is as yet unknown. In the case $a=0$ this problem is called the Dirichlet divisor problem.

It is not obvious in view of existing proofs in the case $a=0$ how to prove our theorem. One of the difficulties is that the "Voronoï series" for $\Delta_{a}(y)$ may diverge for $a \leq-1 / 2$.

Our argument may be generalized to a wider set of real and complex values of $a$ including $a=0$. In the case $a=0$ it clearly gives Preissmann's result mentioned above. But to keep it as simple as possible and referring also to the remarks in Section 4 we assume $-1<a<0$. Moreover, this assumption, being equivalent to $1 / 2<(1-a) / 2<1$, is suggested by the analogy between $\Delta_{a}(x)$ and $E_{(1-a) / 2}(T)$.

It seems difficult to improve the $O$-terms in our theorem. In fact, we believe that they are $\Omega(x)$. For $-1 / 2<a<0$ the $O$-term is $\Omega\left(x^{3 / 4+3 a / 2}\right)$. This can be seen by following the proofs of Theorem 3 in [5] and Theorem 13.6 in [2].

Acknowledgements. I am grateful to Professor Kohji Matsumoto for encouragement and for comments concerning this work. 
2. Proof of the Theorem. We begin by stating our main lemma. Its proof will be given in Sections $3-5$.

Lemma 1. For $-1<a<0, y \geq 1, X \geq y, Z \geq 2 y$ and $y$ not an integer we have

$$
\Delta_{a}(y)=\Delta_{a}(y, X)+R_{a}(y, X, Z)+O\left(y^{-1 / 4+a / 2}\right)+O\left(y^{-1 / 2}\right),
$$

where

$$
\Delta_{a}(y, X)=\frac{1}{\pi \sqrt{2}} y^{1 / 4+a / 2} \int_{1}^{2} \sum_{n \leq u X} \sigma_{a}(n) n^{-3 / 4-a / 2} \cos (4 \pi \sqrt{n y}-\pi / 4) d u
$$

and

$$
R_{a}(y, X, Z)=\frac{1}{2 \pi} \sum_{n \leq Z} \sigma_{a}(n) \int_{1}^{2} \int_{u X}^{\infty} t^{-1} \sin (4 \pi(\sqrt{y}-\sqrt{n}) \sqrt{t}) d t d u .
$$

Remark. The expression $R_{a}(y, X, Z)$ accounts for the jumps of $\Delta_{a}(y)$ at integers. Therefore the easy estimate $R_{a}(y, X, Z) \ll y^{\varepsilon}$-which is not good enough for our purpose - cannot be improved in general. But it can be improved if $y$ is not near an integer and $X$ is large. This way of argument is successful in the case $a=0$ (see [6]) but not here. That is why we need the explicit expression for $R_{a}(y, X, Z)$ as given above.

Suppose that $x \geq 2, x / 2 \leq y \leq x, Z=2 x$ and $y$ is not an integer. We apply Lemma 1 with two different values of $X$, viz. $4 x$ and $x$. We abbreviate just for a moment $R_{a}(y, X, Z)=R_{a}(y, X)$. Then

$$
\begin{aligned}
\Delta_{a}(y)^{2}= & \Delta_{a}(y, 4 x)^{2}+2 \Delta_{a}(y, 4 x) R_{a}(y, 4 x)+R_{a}(y, 4 x)^{2} \\
& +O\left(y^{-1 / 4}\left(\left|\Delta_{a}(y, 4 x)\right|+\left|R_{a}(y, 4 x)\right|\right)+y^{-1 / 2}\right)
\end{aligned}
$$

and

$$
\Delta_{a}(y, 4 x)=\Delta_{a}(y, x)+R_{a}(y, x)-R_{a}(y, 4 x)+O\left(y^{-1 / 4}\right) .
$$

We combine these formulas to obtain

$$
\begin{aligned}
\Delta_{a}(y)^{2}= & \Delta_{a}(y, 4 x)^{2}+2 \Delta_{a}(y, x) R_{a}(y, 4 x)+2 R_{a}(y, x) R_{a}(y, 4 x) \\
& -R_{a}(y, 4 x)^{2}+O\left(y^{-1 / 4}\left(\left|\Delta_{a}(y, 4 x)\right|+\left|R_{a}(y, 4 x)\right|\right)+y^{-1 / 2}\right) .
\end{aligned}
$$

Now we integrate for $y$ and use Cauchy's inequality to obtain

$$
\int_{x / 2}^{x} \Delta_{a}(y)^{2} d y=I_{1}+2 I_{2}+O\left(\sqrt{I_{3} I_{3}^{\prime}}+I_{3}+x^{1 / 4}\left(\sqrt{I}_{1}+\sqrt{I}_{3}\right)+x^{1 / 2}\right),
$$

where

$$
I_{1}=\int_{x / 2}^{x} \Delta_{a}(y, 4 x)^{2} d y, \quad I_{2}=\int_{x / 2}^{x} \Delta_{a}(y, x) R_{a}(y, 4 x, 2 x) d y
$$




$$
I_{3}=\int_{x / 2}^{x} R_{a}(y, 4 x, 2 x)^{2} d y \text { and } I_{3}^{\prime}=\int_{x / 2}^{x} R_{a}(y, x, 2 x)^{2} d y .
$$

Obviously it suffices to prove that

$$
\begin{gathered}
I_{1}= \begin{cases}c_{1}\left(x^{3 / 2+a}-(x / 2)^{3 / 2+a}\right)+O(x) & \text { for }-1 / 2<a<0, \\
c_{2}(x \log x-(x / 2) \log (x / 2))+O(x) & \text { for } a=-1 / 2, \\
O(x) & \text { for }-1<a<-1 / 2,\end{cases} \\
I_{2} \ll x, \\
I_{3} \ll x \quad \text { and } I_{3}^{\prime} \ll x .
\end{gathered}
$$

Pr o of of (2.1). We square out the expression for $\Delta_{a}(y, 4 x)$ given by Lemma 1 and get $I_{1}=I_{10}+I_{11}$, where

$$
\begin{gathered}
I_{10}=\frac{1}{2 \pi^{2}} \int_{x / 2}^{x} y^{1 / 2+a} \sum_{n \leq 8 x} b(n)^{2} \cos ^{2}(4 \pi \sqrt{n y}-\pi / 4) d y \\
I_{11}=\frac{1}{2 \pi^{2}} \int_{x / 2}^{x} y^{1 / 2+a} \sum_{\substack{m, n \leq 8 x \\
m \neq n}} b(m) b(n) \cos (4 \pi \sqrt{m y}-\pi / 4) \cos (4 \pi \sqrt{n y}-\pi / 4) d y \\
b(n)=\sigma_{a}(n) n^{-3 / 4-a / 2} \int_{\max (1, n /(4 x))}^{2} d u .
\end{gathered}
$$

We first prove that $I_{11} \ll x$ (which is acceptable in view of our claim (2.1)). This reduces to showing that $J^{ \pm} \ll x$, where

$$
J^{ \pm}=\int_{x / 2}^{x} y^{1 / 2+a} \sum_{\substack{m, n \leq 8 x \\ m \neq n}} b(m) b(n) e^{4 \pi i(\sqrt{m} \pm \sqrt{n}) \sqrt{y}} d y .
$$

By the second mean value theorem there exist $\xi_{1}$ and $\xi_{2}$ between $x / 2$ and $x$ such that

$$
\begin{aligned}
J^{ \pm} & \ll x^{1+a}\left|\int_{\xi_{1}}^{\xi_{2}} y^{-1 / 2} \sum_{\substack{m, n \leq 8 x \\
m \neq n}} b(m) b(n) e^{4 \pi i(\sqrt{m} \pm \sqrt{n}) \sqrt{y}} d y\right| \\
& \ll x^{1+a} \sum_{j=1}^{2}\left|\sum_{\substack{m, n \leq 8 x \\
m \neq n}} b(m) b(n)(\sqrt{m} \pm \sqrt{n})^{-1} e^{4 \pi i(\sqrt{m} \pm \sqrt{n}) \sqrt{\xi_{j}}}\right| .
\end{aligned}
$$

Trivially $J^{+} \ll x$. Similarly we get trivially $J^{-} \ll x \log x$, but this does not suffice. So, following Preissmann [9], we invoke a generalization of Hilbert's 
inequality, viz. the Montgomery-Vaughan inequality (see [2], (5.34)). We have

$$
\min _{m \neq n}|\sqrt{m}-\sqrt{n}| \gg n^{-1 / 2}
$$

for any positive integer $n$ and it follows that

$$
J^{-} \ll x^{1+a} \sum_{n \leq 8 x} b(n)^{2} n^{1 / 2} \ll x^{1+a} \sum_{n \leq 8 x} \sigma_{a}(n)^{2} n^{-a-1} \ll x
$$

so that $I_{11} \ll x$ as claimed.

Consider $I_{10}$. Since $\cos ^{2}(4 \pi \sqrt{n y}-\pi / 4)=(1+\sin (8 \pi \sqrt{n y})) / 2$, and

$$
\sum_{n \leq 8 x} b(n)^{2} \int_{x / 2}^{x} y^{1 / 2+a} \sin (8 \pi \sqrt{n y}) d y \ll x^{1+a}
$$

(see [10], Lemma 4.3), we have

$$
I_{10}=\frac{1}{(6+4 a) \pi^{2}}\left(x^{3 / 2+a}-(x / 2)^{3 / 2+a}\right) \sum_{n \leq 8 x} b(n)^{2}+O\left(x^{1+a}\right) .
$$

Here

$$
\sum_{n \leq 8 x} b(n)^{2}=\sum_{n \leq x} \sigma_{a}(n)^{2} n^{-3 / 2-a}+O\left(x^{-1 / 2-a}\right),
$$

which is $O\left(x^{-1 / 2-a}\right)$ for $-1<a<-1 / 2$ so that $I_{10} \ll x$ in this case. For $-1 / 2<a<0$ we have (see [10], (1.3.3))

$$
\sum_{n \leq x} \sigma_{a}(n)^{2} n^{-3 / 2-a}=\zeta(3 / 2-a) \zeta(3 / 2+a) \zeta(3 / 2)^{2} \zeta(3)^{-1}+O\left(x^{-1 / 2-a}\right)
$$

so that $I_{10}=c_{1}\left(x^{3 / 2+a}-(x / 2)^{3 / 2+a}\right)+O(x)$ in this case. In the remaining case $a=-1 / 2$ we use Perron's formula to obtain

$$
\sum_{n \leq x} \sigma_{a}(n)^{2} n^{-1}=\zeta(3 / 2)^{2} \zeta(2) \zeta(3)^{-1} \log x+O(1) .
$$

Since $\zeta(2)=\pi^{2} / 6$ we conclude that $I_{10}=c_{2}(x-x / 2) \log x+O(x)$ in this case. This completes the proof of (2.1).

Proof of (2.2). By the second mean value theorem there exists $\xi$ between $x / 2$ and $x$ such that

$$
I_{2}=x^{3 / 4+a / 2} \int_{\xi}^{x} y^{-3 / 4-a / 2} \Delta_{a}(y, x) R_{a}(y, X, 2 x) d y,
$$

where $X=4 x$. Lemma 1 then gives

$$
I_{2} \ll x^{3 / 4+a / 2} \sum_{m \leq 2 x} \sigma_{a}(m) m^{-3 / 4-a / 2} \sum_{n \leq 2 x} \sigma_{a}(n)|J(m, n, X)|,
$$


where

$J(m, n, X)$

$$
=\int_{\xi}^{x} y^{-1 / 2} \cos (4 \pi \sqrt{m y}-\pi / 4) \int_{1}^{2} \int_{u X}^{\infty} t^{-1} \sin (4 \pi(\sqrt{y}-\sqrt{n}) \sqrt{t}) d t d u d y .
$$

Then for the proof of (2.2) it clearly suffices to show that

$$
\sum_{n \leq 2 x} \sigma_{a}(n)|J(m, n, X)| \ll 1 .
$$

For $Y \geq X$ we have

$$
J(m, n, X)-J(m, n, Y) \ll x^{-1 / 2} \max _{y \in\{\xi, x\}} \min \left(1,|y-n|^{-1}\right)
$$

uniformly in $Y$, since

$$
\begin{aligned}
\int_{u X}^{u Y} t^{-1} e^{-4 \pi i \sqrt{n t}} \int_{\xi}^{x} y^{-1 / 2} e^{4 \pi i(\sqrt{t} \pm \sqrt{m}) \sqrt{y}} d y d t \\
\ll \max _{y \in\{\xi, x\}}\left|\int_{u X}^{u Y} t^{-1}(\sqrt{t} \pm \sqrt{m})^{-1} e^{-4 \pi i(\sqrt{n t}-(\sqrt{t} \pm \sqrt{m}) \sqrt{y})} d t\right| \\
=\max _{y \in\{\xi, x\}}\left|\int_{u X}^{u Y} t^{-1}(\sqrt{t} \pm \sqrt{m})^{-1} e^{4 \pi i(\sqrt{y}-\sqrt{n}) \sqrt{t}} d t\right| \\
\ll \max _{y \in\{\xi, x\}} \min \left(X^{-1 / 2}, X^{-1}|\sqrt{y}-\sqrt{n}|^{-1}\right) .
\end{aligned}
$$

In the last step we applied Lemma 4.3 in [10], and made use of the fact that $m \leq 2 x=X / 2$. (At this point one can see why Lemma 1 was applied with two different values of $X$.) On the other hand, $\lim _{Y \rightarrow \infty} J(m, n, Y)=0$ by applying the same lemma to the innermost integral. Now (2.4) follows easily.

Proof of (2.3). We need the following lemma, the proof of which is a simple application of partial integration.

Lemma 2. For $X \geq 1$ and any real $k$ we have

$$
\int_{1}^{2} \int_{u X}^{\infty} t^{-1} \sin (k \sqrt{t}) d t d u \ll \min \left(1, X^{-1} k^{-2}\right) .
$$

Lemma 1, Lemma 2 and Cauchy's inequality give 


$$
\begin{aligned}
I_{3} & \ll \int_{x / 2}^{x}\left(\sum_{n \leq 2 x} \sigma_{a}(n) \min \left(1,(n-y)^{-2}\right)\right)^{2} d y \\
& \ll \int_{x / 2}^{x} \sum_{n \leq 2 x} \sigma_{a}(n)^{2} \min \left(1,(n-y)^{-2}\right) d y \\
& \ll \sum_{n \leq 2 x} \sigma_{a}(n)^{2} \int_{x / 2}^{x} \min \left(1,(n-y)^{-2}\right) d y \\
& \ll \sum_{n \leq 2 x} \sigma_{a}(n)^{2} \ll x .
\end{aligned}
$$

The integral $I_{3}^{\prime}$ is estimated similarly and (2.3) follows.

3. Analytic continuation. In the following sections we prove Lemma 1.

Let $z$ be a complex variable and let $p$ be a real variable, which will eventually tend to $\infty$. Let $w$ be a sufficiently many (three will suffice) times continuously differentiable function supported on the interval $[-2 / 3,2 / 3]$ such that $w(v)=1$ for $v \in[-1 / 3,1 / 3]$. It is clear that the function $z \mapsto$ $\Delta_{z}(y)$ is entire. Hence, defining

$$
\Delta_{z, p}(y)=p \int_{-\infty}^{\infty} w(v) e^{-\pi(p v)^{2}}(1+v)^{1 / 2-z} \Delta_{z}\left(y(1+v)^{2}\right) d v,
$$

the function $z \mapsto \Delta_{z, p}(y)$ is entire. We define

$B_{z}(t)=\sin (\pi z / 2) J_{1+z}(4 \pi \sqrt{t})+\cos (\pi z / 2)\left(Y_{1+z}(4 \pi \sqrt{t})+(2 / \pi) K_{1+z}(4 \pi \sqrt{t})\right)$ in the usual notation of Bessel functions. Oppenheim [7] has proved that

$$
\Delta_{z}(y)=-y^{(1+z) / 2} \sum_{n=1}^{\infty} \sigma_{z}(n) n^{-(1+z) / 2} B_{z}(n y)
$$

for $-1 / 2<z<0$. The series here is boundedly convergent in any finite $y$-subinterval of $(0, \infty)$, as shown by Hafner [1]. Hence we may integrate term-by-term to obtain

$$
\begin{aligned}
\Delta_{z, p}(y)= & -p y^{(1+z) / 2} \sum_{n=1}^{\infty} \sigma_{z}(n) n^{-(1+z) / 2} \\
& \times \int_{-\infty}^{\infty} w(v) e^{-\pi(p v)^{2}}(1+v)^{3 / 2} B_{z}\left(n y(1+v)^{2}\right) d v .
\end{aligned}
$$

We now only know that (3.2) holds for real values of $z$ satisfying $-1 / 2<$ $z<0$. 
Consider the expression

$$
p y^{-1 / 4+z / 2} \sum_{n=1}^{\infty} \sigma_{z}(n) n^{-5 / 4-z / 2} \int_{-\infty}^{\infty} w(v) e^{-\pi(p v)^{2}} h_{z}\left(n y(1+v)^{2}\right) d v
$$

where

$$
h_{z}(t)=\frac{1}{\pi \sqrt{2}}\left(\sqrt{t} \cos (4 \pi \sqrt{t}-\pi / 4)-\frac{4 z^{2}+8 z+3}{32 \pi} \sin (4 \pi \sqrt{t}-\pi / 4)\right) .
$$

By partial integration (this is where we need the function $w$ ) and the familiar formula

$$
\int_{-\infty}^{\infty} e^{A v-B v^{2}} d v=\sqrt{\pi / B} e^{A^{2} /(4 B)} \quad(\Re(B)>0)
$$

(see e.g. [2], (A.38)), we get

$$
p \int_{-\infty}^{\infty} w(v) e^{-\pi(p v)^{2}+4 \pi i v \sqrt{n y}} d v=e^{-4 \pi n y / p^{2}}+O\left((n y)^{-3 / 2} e^{-p}\right)
$$

and (using (3.4))

$$
\begin{aligned}
p \int_{-\infty}^{\infty} w(v) v e^{-\pi(p v)^{2}+4 \pi i v \sqrt{n y}} d v & \\
= & 2 i(n y)^{1 / 2} p^{-2} e^{-4 \pi n y / p^{2}}+O\left((n y)^{-1} e^{-p}\right) .
\end{aligned}
$$

Let $\mathcal{C}$ be a compact subset of $\mathcal{D}=\{z \mid-3 / 2<\Re(z)<3 / 2\}$. It follows that the series in (3.3) is absolutely and uniformly convergent in $\mathcal{C}$ and hence that the expression (3.3) defines a holomorphic function $z \mapsto \Delta_{z, p}^{*}(y)$, say, in $\mathcal{D}$.

By (3.2) and (3.3) we get a series representing $\Delta_{z, p}(y)-\Delta_{z, p}^{*}(y)$ for $-1 / 2<z<0$. It has holomorphic terms in $\mathcal{D}$. It is absolutely and uniformly convergent and $O\left(\left|y^{-3 / 4+z / 2}\right|\right)$ in $\mathcal{C}$, since, by well-known asymptotic formulas for Bessel functions (see [12], Sec. 7.21, 7.23),

$$
t^{3 / 4} B_{z}(t)+h_{z}(t) \ll t^{-1 / 2}
$$

uniformly in $\mathcal{C}$. Hence it is holomorphic and represents $\Delta_{z, p}(y)-\Delta_{z, p}^{*}(y)$ in the whole $\mathcal{D}$, and we get $\Delta_{z, p}(y)-\Delta_{z, p}^{*}(y) \ll\left|y^{-3 / 4+z / 2}\right|$ for $z$ in $\mathcal{D}$.

Finally, we evaluate $\Delta_{a, p}^{*}(y)$ using (3.4) and (3.5) and conclude that

$$
\Delta_{a, p}(y)=\Delta_{a, p}^{(1)}(y)+\Delta_{a, p}^{(2)}(y)+O\left(\left|y^{-3 / 4+a / 2}\right|\right),
$$

where

$$
\begin{aligned}
\Delta_{a, p}^{(1)}(y)= & \frac{1}{\pi \sqrt{2}} y^{1 / 4+a / 2} \sum_{n=1}^{\infty} \sigma_{a}(n) n^{-3 / 4-a / 2} e^{-4 \pi n y / p^{2}} \\
& \times \cos (4 \pi \sqrt{n y}-\pi / 4)
\end{aligned}
$$




$$
\begin{aligned}
\Delta_{a, p}^{(2)}(y)= & y^{-1 / 4+a / 2} \sum_{n=1}^{\infty} \sigma_{a}(n)\left(c_{3}-\sqrt{2} \pi^{-1} n y / p^{2}\right) n^{-5 / 4-a / 2} \\
& \times e^{-4 \pi n y / p^{2}} \sin (4 \pi \sqrt{n y}-\pi / 4)
\end{aligned}
$$

and $c_{3}=-\left(4 a^{2}+8 a+3\right) /\left(32 \pi^{2} \sqrt{2}\right)$.

Re marks. The quantity $c_{3}$ vanishes at $a=-1 / 2$. The implied constant in (3.6) does not depend on $p$ and the formula is valid for any $a$ in $\mathcal{D}$. This range can be further extended by replacing $-h_{z}(t)$ with a sharper approximation of $t^{3 / 4} B_{z}(t)$.

\section{Lemmata}

Lemma 3. For $X \geq 1, Y \geq X, V>0, l$ fixed and any real $k$ we have

$$
\int_{1}^{2} \int_{u X}^{u Y} t^{-l} e^{-t / V+i k \sqrt{t}} d t d u \ll \begin{cases}X^{-l} \min \left(V, k^{-2}\right) & \text { for } l \geq 0, \\ X^{-l} \min \left(X, k^{-2}\right) & \text { for } l>1 .\end{cases}
$$

Proof. Partial integration gives $O\left(X^{-l} k^{-2}\right)$ if $k \neq 0$. The alternative estimates are trivial.

Lemma 4. For $-3 / 2<a<3 / 2$ we have

$$
\int_{0}^{y} \Delta_{a}(v) d v=c_{4}+y^{3 / 4+a / 2} \sum_{n=1}^{\infty} \sigma_{a}(n) n^{-5 / 4-a / 2} g(n y)+O\left(y^{-3 / 4+a / 2}\right),
$$

where

$$
g(t)=\sum_{\nu=0}^{2} e_{\nu} t^{-\nu / 2} \cos (4 \pi \sqrt{t}+\pi / 4+\pi \nu / 2),
$$

$e_{0}=1 /\left(2 \pi^{2} \sqrt{2}\right), e_{1}, e_{2}$ and $c_{4}$ may depend on a only and the series here is uniformly convergent on any finite closed subinterval of $(0, \infty)$.

Pr o of. The lemma is based on Theorem B and Lemma 2.1 of Hafner [1]. See also Section 2 of [5].

LEMma 5. For $-1<a<1 / 2$ we have

$$
\int_{0}^{y} \Delta_{a}(v) d v \ll y^{3 / 4+a / 2}+y^{1 / 2} \log y .
$$

Proof. The integral is $O\left(y^{3 / 4+a / 2}\right)$ for $-1 / 2<a<1 / 2$ by Lemma 4 , whereas the case $-1<a \leq-1 / 2$ is covered by Lemma 2 of [5].

Remarks. The restriction $-3 / 2<a<3 / 2$ in Lemma 4 is essential. Since the number $r$ in Hafner's Definition 1.1 is real, our a must be real. It is, however, possible to generalize Lemma 4 to complex values of $a$. The 
assumption $a>-1$ in Lemma 5 is not essential, but we have to accept it because it occurs in Lemma 2 of [5].

5. Transformation. The idea now is to truncate the series in (3.7) and (3.8), transform the remainder using Lemma 4 and then let $p \rightarrow \infty$ along a suitable sequence. The constants implied by the symbols $O$ and $\ll$ will be independent of $p$.

We define

$$
f_{p}(t)=t^{-3 / 4-a / 2} e^{-4 \pi t y / p^{2}} \cos (4 \pi \sqrt{t y}-\pi / 4) .
$$

Let $1 \leq u \leq 2$. We have

$$
\begin{aligned}
\sum_{n>u X} \sigma_{a}(n) f_{p}(n)= & -\int_{u X}^{\infty} f_{p}^{\prime}(t)\left(D_{a}(t)-D_{a}(u X)\right) d t \\
= & -\int_{u X}^{\infty} f_{p}^{\prime}(t)\left[\zeta(1-a) v+\frac{\zeta(1+a)}{1+a} v^{1+a}\right]_{v=u X}^{t} d t \\
& -\int_{u X}^{\infty} f_{p}^{\prime}(t)\left(\Delta_{a}(t)-\Delta_{a}(u X)\right) d t \\
= & \int_{u X}^{\infty} f_{p}(t)\left(\zeta(1-a)+\zeta(1+a) t^{a}\right) d t \\
& -f_{p}(u X) \Delta_{a}(u X)+\int_{u X}^{\infty} f_{p}^{\prime \prime}(t) \int_{u X}^{t} \Delta_{a}(v) d v d t .
\end{aligned}
$$

Hence

$$
\int_{1}^{2} \sum_{n>u X} \sigma_{a}(n) f_{p}(n) d u=S_{1}(p)+S_{2}(p)+\lim _{Y \rightarrow \infty} S_{3}(p, Y),
$$

where

$$
\begin{aligned}
S_{1}(p) & =-\int_{1}^{2} f_{p}(u X) \Delta_{a}(u X) d u, \\
S_{2}(p) & =\int_{1}^{2} \int_{u X}^{\infty} f_{p}(t)\left(\zeta(1-a)+\zeta(1+a) t^{a}\right) d t d u, \\
S_{3}(p, Y) & =\int_{1}^{2} \int_{u X}^{u Y} f_{p}^{\prime \prime}(t) \int_{u X}^{t} \Delta_{a}(v) d v d t d u .
\end{aligned}
$$

We claim that

$$
S_{1}(p) \ll y^{-1 / 2}, \quad S_{2}(p) \ll y^{-1 / 2} .
$$


Concerning $S_{2}(p)$ this is clear, since Lemma 3 gives $S_{2}(p) \ll y^{-1} X^{-3 / 4-a / 2}$. Consider then $S_{1}(p)$. We have

$$
\begin{aligned}
S_{1}(p) & =-X^{-1}\left[f_{p}(t) \int_{0}^{t} \Delta_{a}(v) d v\right]_{t=X}^{2 X}+X^{-1} \int_{X}^{2 X} f_{p}^{\prime}(t) \int_{0}^{t} \Delta_{a}(v) d v d t \\
& =X^{-1} S_{11}+X^{-1} S_{12},
\end{aligned}
$$

say. Lemma 5 gives $S_{11} \ll 1+X^{-1 / 4-a / 2} \log X$, which is acceptable. Lemma 4 gives

$$
\begin{aligned}
S_{12} & \ll \\
& \sum_{n=1}^{\infty} n^{-3 / 4}\left|\int_{X}^{2 X} f_{p}^{\prime}(t) t^{3 / 4+a / 2} g(n t) d t\right|+\int_{X}^{2 X}\left|f_{p}^{\prime}(t)\right| t^{-3 / 4+a / 2} d t \\
& +\left|\int_{X}^{2 X} f_{p}^{\prime}(t) d t\right| \\
= & S_{121}+S_{122}+S_{123},
\end{aligned}
$$

say. By (5.1) we have

$$
\begin{aligned}
f_{p}^{\prime}(t)= & t^{-3 / 4-a / 2}\left(-2 \pi y^{1 / 2} t^{-1 / 2} \sin (4 \pi \sqrt{t y}-\pi / 4)\right. \\
& \left.+\left(c_{5} y p^{-2}+c_{6} t^{-1}\right) \cos (4 \pi \sqrt{t y}-\pi / 4)\right) e^{-4 \pi t y / p^{2}}
\end{aligned}
$$

where $c_{5}=-4 \pi$ and $c_{6}=-3 / 4-a / 2$, so that

$$
\int_{X}^{2 X} f_{p}^{\prime}(t) t^{3 / 4+a / 2} g(n t) d t \ll y^{1 / 2} \min \left(X^{1 / 2},|\sqrt{n}-\sqrt{y}|^{-1}\right)
$$

either trivially or by Lemma 4.3 of [10]. Hence $S_{121} \ll y^{1 / 4} \log y+X^{1 / 2} y^{-1 / 4}$. Finally, it is plain that $S_{122} \ll y^{1 / 2} X^{-1}, S_{123} \ll X^{-3 / 4-a / 2}$ and (5.3) has been proved.

Consider $S_{3}(p, Y)$. We apply Lemma 4 and integrate term-by-term to get

$$
\begin{aligned}
S_{3}(p, Y)= & \sum_{n=1}^{\infty} \sigma_{a}(n) n^{-5 / 4-a / 2} \int_{1}^{2} \int_{u X}^{u Y} f_{p}^{\prime \prime}(t)\left[y^{3 / 4+a / 2} g(n y)\right]_{y=u X}^{t} d t d u \\
& +O\left(X^{-3 / 4+a / 2} \int_{X}^{2 Y}\left|f_{p}^{\prime \prime}(t)\right| d t\right) .
\end{aligned}
$$

The $O$-term here is $O\left(y X^{-3 / 2}\right)$, since (5.4) implies that $f_{p}^{\prime \prime}(t) \ll y t^{-7 / 4-a / 2}$. Then we integrate by parts and note that the integrated term is

$$
\int_{1}^{2} f_{p}^{\prime}(u Y)\left(\int_{u X}^{u Y} \Delta_{a}(v) d v+O\left(X^{-3 / 4+a / 2}\right)\right) d u \ll_{y} Y^{2} e^{-Y y / p^{2}} .
$$


Hence

$$
\begin{aligned}
S_{3}(p, Y)= & -\sum_{n=1}^{\infty} \sigma_{a}(n) n^{-5 / 4-a / 2} \int_{1}^{2} \int_{u X}^{u Y} f_{p}^{\prime}(t)\left(t^{3 / 4+a / 2} g(n t)\right)^{\prime} d t d u \\
& +O\left(y X^{-3 / 2}\right)+O_{y}\left(Y^{2} e^{-Y y / p^{2}}\right) .
\end{aligned}
$$

We have

$$
\begin{aligned}
\left(t^{3 / 4+a / 2} g(n t)\right)^{\prime}= & t^{3 / 4+a / 2}\left((\pi \sqrt{2})^{-1} n^{1 / 2} t^{-1 / 2} \cos (4 \pi \sqrt{n t}-\pi / 4)\right. \\
& \left.+c_{7} t^{-1} \sin (4 \pi \sqrt{n t}-\pi / 4)+O\left(n^{-1 / 2} t^{-3 / 2}\right)\right),
\end{aligned}
$$

where $c_{7}$ may depend on $a$ only. Hence, by (5.4), Lemma 3 and using the formula $\sin \alpha \cos \beta=(\sin (\alpha+\beta)+\sin (\alpha-\beta)) / 2$, we get (assuming that $\left.p^{2}>X y\right)$

$$
\begin{aligned}
& \int_{1}^{2} \int_{u X}^{u Y} f_{p}^{\prime}(t)\left(t^{3 / 4+a / 2} g(n t)\right)^{\prime} d t d u \\
&=-(1 / \sqrt{2})(n y)^{1 / 2} I(n, p, Y) \\
&+ O\left((y+n)^{1 / 2} X^{-3 / 2} \min \left(X,(\sqrt{n}-\sqrt{y})^{-2}\right)\right) \\
&+O\left(y^{1 / 2} X^{-1} n^{-1 / 2}\right),
\end{aligned}
$$

where

$$
I(n, p, Y)=\int_{1}^{2} \int_{u X}^{u Y} t^{-1} e^{-4 \pi y t / p^{2}} \sin (4 \pi(\sqrt{y}-\sqrt{n}) \sqrt{t}) d t d u .
$$

For $n>Z$ we have $I(n, p, Y) \ll(X n)^{-1}$ by Lemma 3 , since $Z \geq 2 y$ by assumption. Hence

$$
\begin{aligned}
S_{3}(p, Y)= & \frac{1}{\sqrt{2}} y^{1 / 2} \sum_{n \leq Z} \sigma_{a}(n) n^{-3 / 4-a / 2} I(n, p, Y) \\
& +O_{y}\left(Y^{2} e^{-Y y / p^{2}}\right)+O\left(y^{1 / 2} X^{-1}\right)+O\left(y^{-3 / 4-a / 2+\varepsilon} X^{-1 / 2}\right)
\end{aligned}
$$

for any $\varepsilon>0$.

We combine (3.7), (5.2), (5.3) and (5.5). This gives

$$
\begin{aligned}
\Delta_{a, p}^{(1)}(y)= & \frac{1}{\pi \sqrt{2}} y^{1 / 4+a / 2} \int_{1}^{2} \sum_{n \leq u X} \sigma_{a}(n) f_{p}(n) d u \\
& +\frac{1}{2 \pi} y^{3 / 4+a / 2} \sum_{n \leq Z} \sigma_{a}(n) n^{-3 / 4-a / 2} \lim _{Y \rightarrow \infty} I(n, p, Y) \\
& +O\left(y^{-1 / 4+a / 2}\right) .
\end{aligned}
$$


Concerning $\Delta_{a, p}^{(2)}(y)$, as defined by (3.8), we argue similarly with $X$ replaced by $y$ and estimate trivially the contribution of the terms with $n \ll y$. Here it is to be noted that $c_{3}=0$ at $a=-1 / 2$. The result is that

$$
\Delta_{a, p}^{(2)}(y) \ll y^{-1 / 4+a / 2}+y^{-1 / 2} .
$$

Next, we show that

$$
\lim _{p \rightarrow \infty} \Delta_{a, p}(y)=\Delta_{a}(y)
$$

unless $y$ is an integer. First of all we have

$$
p \int_{-\infty}^{\infty} w(v) e^{-\pi(p v)^{2}}(1+v)^{1 / 2-a} d v=1+O\left(p^{-1}\right) .
$$

It follows that (see (3.1))

$$
\begin{aligned}
\Delta_{a, p}(y)- & \Delta_{a}(y) \\
= & p \int_{-\infty}^{\infty} w(v) e^{-\pi(p v)^{2}}(1+v)^{1 / 2-a}\left(\Delta_{a}\left(y(1+v)^{2}\right)-\Delta_{a}(y)\right) d v \\
& +O\left(\left|\Delta_{a}(y)\right| / p\right) \\
\ll & \int_{-2 / 3}^{2 / 3} e^{-\pi(p v)^{2}}\left|\Delta_{a}\left(y(1+v)^{2}\right)-\Delta_{a}(y)\right| d v+y p^{-1} \\
\ll & \int_{0}^{2 / 3} e^{-\pi(p v)^{2}}\left(y v+\sum_{|n-y| \leq 2 y v} \sigma_{a}(n)\right) d v+y p^{-1} \\
\ll & \int_{0}^{2 p / 3} e^{-\pi v^{2}} \sum_{|n-y| \leq 2 y v / p} \sigma_{a}(n) d v+y p^{-1} .
\end{aligned}
$$

Clearly this tends to zero as $p \rightarrow \infty$ unless $y$ is an integer, as claimed.

We combine (3.6), (5.6)-(5.8) and let $p \rightarrow \infty$. This gives

$$
\begin{aligned}
\Delta_{a}(y)= & \Delta_{a}(y, X)+\frac{1}{2 \pi} y^{3 / 4+a / 2} \sum_{n \leq Z} \sigma_{a}(n) n^{-3 / 4-a / 2} \lim _{p \rightarrow \infty} \lim _{Y \rightarrow \infty} I(n, p, Y) \\
& +O\left(y^{-1 / 4+a / 2}\right)+O\left(y^{-1 / 2}\right)
\end{aligned}
$$

unless $y$ is an integer. It is easy to show that

$$
\lim _{p \rightarrow \infty} \lim _{Y \rightarrow \infty} I(n, p, Y)=\int_{1}^{2} \int_{u X}^{\infty} t^{-1} \sin (4 \pi(\sqrt{y}-\sqrt{n}) \sqrt{t}) d t d u .
$$


Finally, we replace $n^{-3 / 4-a / 2}$ in the sum by $y^{-3 / 4-a / 2}$. By Lemma 2 , this produces a term $O\left(y^{-1 / 4+a / 2}\right)$ to the whole expression. The proof of Lemma 1 is thus complete.

\section{References}

[1] J. L. Hafner, On the representation of the summatory functions of a class of arithmetical functions, in: Analytic Number Theory, M. I. Knopp (ed.), Lecture Notes in Math. 899, Springer, 1981, 148-165.

[2] A. Ivić, The Riemann Zeta-Function, Wiley, New York, 1985.

[3] I. Kiuchi, On an exponential sum involving the arithmetic function $\sigma_{a}(n)$, Math. J. Okayama Univ. 29 (1987), 193-205.

[4] K. Matsumoto and T. Meurman, The mean square of the Riemann zeta-function in the critical strip II, Acta Arith. 68 (1994), 369-382.

[5] - - - The mean square of the Riemann zeta-function in the critical strip III, ibid. 64 (1993), 357-382.

[6] T. Meurman, On the mean square of the Riemann zeta-function, Quart. J. Math. Oxford (2) 38 (1986), 337-343.

[7] A. Oppenheim, Some identities in the theory of numbers, Proc. London Math. Soc. (2) 26 (1927), 295-350.

[8] Y.-F. S. Pétermann, Divisor problems and exponent pairs, Arch. Math. (Basel) 50 (1988), 243-250.

[9] E. Preissmann, Sur la moyenne quadratique du terme de reste du problème du cercle, C. R. Acad. Sci. Paris Sér. I 306 (1988), 151-154.

[10] E. C. Titchmarsh, The Theory of the Riemann Zeta-Function, Oxford University Press, Oxford, 1951.

[11] K.-C. Tong, On divisor problems III, Acta Math. Sinica 6 (1956), 515-541.

[12] G. N. Watson, A Treatise on the Theory of Bessel Functions, 2nd ed., Cambridge University Press, Cambridge, 1944.

Department of Mathematics

University of Turku

FIN-20500 Turku, Finland 